\title{
Changes in nerve conduction in experimental allergic neuritis
}

\author{
B. G. CRAGG AND P. K. THOMAS ${ }^{1}$ \\ From the Department of Anatomy, University College, London
}

Experimental allergic neuritis was the term given by Waksman and Adams (1955) to the peripheral neuropathy induced in animals by the inoculation of homologous or heterologous peripheral nerve tissue combined with adjuvants. Lesions occur in the spinal nerve roots, spinal ganglia, and peripheral nerves in the form of focal perivascular accumulations of inflammatory cells related to areas of myelin destruction with relative sparing of the axons. Although the histological changes have been described in detail, the accompanying alterations in nerve conduction have not yet been fully investigated. Kaeser and Lambert (1962) and Kaeser (1962) showed that nerve fibres affected by this process may either cease to conduct or exhibit a marked reduction in conduction velocity. The present investigation reports some further observations on the alterations in nerve conduction in allergic neuritis, together with a consideration of their explanation in terms of the associated histological changes.

Experimental allergic neuritis shows certain similarities to acute infectious polyneuritis in man (Waksman and Adams, 1955) and it was hoped that the study of the former condition might also throw some light on the alterations in nerve conduction observed in human polyneuritis (Cerra and Johnson, 1961 ; Lambert, 1962; Bannister and Sears, 1962).

\section{METHODS}

ALLERGIC NEURITIS The method of Waksman and Adams (1955) was used in unselected adult male and female guinea-pigs. A heterologous antigen was prepared from the sciatic nerve trunks of two freshly killed rabbits under sterile conditions. Using sterile apparatus throughout, the nerves were cut into short lengths which were placed on a freezing microtome and cut into a mush at a nominal advancement of $5 \mu$. The mush was frozen and recut twice to make a fine brei which was then weighed (about 1 g.). One $10 \mathrm{ml}$. vial of Freund's complete adjuvant (Difco) was opened and $30 \mathrm{mg}$. of killed dried Mycobacterium butyricum (Difco) added. After shaking the adjuvants, a volume (in ml.) equal to one and a half times

'Present address: Institute of Neurology, Queen Square, London, W.C.1. the weight of sciatic nerve brei (in g.) was added to then latter, and the whole emulsified by five minutes' treat- $\mathrm{D}$ ment with a fast electric blender. The resulting emulsion was nearly solid, and did not spread when a small dropes was placed on a water surface. This emulsion contained approximately $0.4 \mathrm{~g}$. sciatic nerve, $0.5 \mathrm{ml}$. Bayol F, $0.1 \mathrm{ml} \cdot$ Arlacel $\mathrm{A}$, and $2 \mathrm{mg}$. bacilli per millilitre. Guinea-pigs $\vec{\omega}$ were injected with $0.01 \mathrm{ml}$. of emulsion intradermallys in each footpad ( $0.04 \mathrm{ml}$. per animal) once only.

ELECTRICAL RECORDING Both peroneal nerves and in some instances the tibial nerves were dissected and removed under Nembutal (pentobarbital sodium) anaesthesia. After a brief wash in saline, the nerve was arranged on electrodes placed at intervals of $5 \mathrm{~mm}$. in a bath of liquid paraffin oil that was maintained at a fixed temperature, usually $38^{\circ} \mathrm{C}$. For measurements of conduction velocity, rectangular stimulating pulses of $0.1 \mathrm{~m} . \mathrm{sec} \frac{\mathrm{O}}{\mathrm{O}}$ duration were delivered through an isolating transforner= to the electrodes at one end of the nerve trunk, usuaty the distal end. After adjusting the stimulus voltage elicit a maximal A fibre potential, the recurrence rate was set at $1 / \mathrm{sec}$, and the stimulus parameters were not changed thereafter. The cathode follower input leads were connected to electrodes at the other end of the nerve $\bar{\partial}$ and after each photograph had been taken these leadso were moved to electrodes $5 \mathrm{~mm}$. nearer to the stimulatedळ end of the nerve. One of the electrodes between the positions of stimulation and the recording was earthed. $\overline{\bar{O}}$

The oscilloscope traces were photographed at full size on recording paper and each photograph contained $10 \mathrm{kc} . / \mathrm{sec}$. marker that was locked both to the trace and? to the stimulator, so that successive traces and markers could be superimposed in each photograph. The latency? of a point halfway up the rising face of the action potential was plotted against conduction distance, and con- 3 duction velocity determined from the best straight line drawn by eye. The response to repetitive stimulation was? also assessed. For high frequency stimulation, a standard tetanus of 368 stimuli per second for two minutes was? employed.

For comparision with the nerves of animals affected byo allergic neuritis, electrical recordings were made from the peroneal, saphenous, sural, and vagus nerves of normabs animals, from the peroneal nerve during Wallerian degeneration after nerve section, and also from this nerve during regeneration after a localized crush injury. Detailsw of the experimental procedures employed are given with the description of the results obtained. 
HISTOLOGY The nerves were washed in saline after removal from the paraffin bath and blotted on filter paper. Short lengths from the distal and proximal ends were attached to card frames and fixed in Flemming's solution. The remaining lengths of nerve trunk were attached to card frames and fixed in $10 \%$ neutral formol saline. The material fixed in Flemming's solution was embedded in paraffin wax and sectioned transversely at $5 \mu$ to be stained by the modified Weigert method described by Gutmann and Sanders (1943) for the examination of the myelin sheaths. Because of the difficulty in following individual fibres for long distances in longitudinal sections, portions of the formol-fixed material were stained with osmium tetroxide and teased apart in glycerine by the method described by Thomas (1955) in order to examine single nerve fibres. Another part of the formol-fixed material was stained by the Marchi method for degenerating myelin and, after paraffin embedding, by the GleesMarsland silver impregnation method for the examination of axons. Cellular infiltration was studied in sections cut longitudinally at $25 \mu$ on a freezing microtome with buffered cresyl violet at $p \mathrm{H} 4.0$ or in $12 \mu$ longitudinal paraffin sections stained by haematoxylin and eosin.

\section{RESULTS}

INCIDENCE OF NEUROPATHY Fifty-nine guinea-pigs were treated and of these, 15 appeared to be completely unaffected. With the experimental procedure employed, paralysis, which was most noticeable in the hind limbs, usually developed at about three weeks after injection and reached a maximum at four to five weeks. Animals that showed marked weakness or paralysis did not survive more than eight weeks, but others that were only slightly affected recovered completely within 10 weeks of injection. Of the 44 affected animals, 34 died before biopsy and 10 that showed weakness of one or both hind limbs were biopsied. The small number of animals examined electrically is explained by the fact that it was difficult to predict the course of the illness, and in an attempt to obtain recordings from more severely affected guinea-pigs many unexpected deaths occurred.

Six animals were biopsied between 33 and 55 days after injection during the stage of paralysis. Four animals that had been mildly affected and had recovered were biopsied between 91 and 113 days after injection. However, precise correlations between the electrical findings and the degree of paralysis are not justifiable as the animals may develop lesions within the central nervous system as well as in the nerve roots and peripheral nerve trunks. Moreover, slight degrees of paralysis in the limbs are difficult to detect in the guinea-pig.

ELECTRICAL OBSERVATIONS On the basis of the electrical findings, it was possible to divide the results into three groups. In some nerves, maximal conduction velocity did not differ significantly from normal, and the action potentials were normal or showed only minor abnormalities. In other nerves, the action potentials consisted of two distinct peaks corresponding to damaged and undamaged fibres. Finally, some nerves gave only abnormal responses.

There were nine nerves in the first group. Most of these nerves were obtained from the less severely affected animals and all those from the recovered animals fell into this category. The maximal conduction velocities of these nerves ranged from 38 to 69 $\mathrm{m}$. $/ \mathrm{sec}$. with a mean of $58 \mathrm{~m}$. $/ \mathrm{sec}$. and a standard deviation of $11.5 \mathrm{~m} . / \mathrm{sec}$. In 10 normal guinea-pig peroneal nerves, the fastest fibres were found to have a velocity range of 60 to $72 \mathrm{~m}$./sec. with a mean of $64.6 \mathrm{~m}$. $/ \mathrm{sec}$. and a standard deviation of $3.9 \mathrm{~m}$. $/ \mathrm{sec}$. This mean velocity did not differ significantly from that of the nine nerves from the affected animals ( $P=0.1$ by Student's $t$ test), although the standard deviation was only one third of that of the affected nerves. Kaeser and Lambert (1962) found a range of 48 to $63 \mathrm{~m}$. $/ \mathrm{sec}$. with a mean of $54 \mathrm{~m}$. $/ \mathrm{sec}$. for the fastest motor nerve fibres to the muscles of the second interosseous space of the foot of normal guinea-pigs measured in the sciatic nerve between the hip and ankle. The muscles at the periphery of the limb are innervated by nerve fibres of smaller diameter than those supplying more proximal muscles (Fernand and Young, 1951). This, and the fact that the present observations were made on a mixed nerve trunk, presumably accounts for the slightly lower value obtained by Kaeser and Lambert.

Recordings of the action potential at various conduction distances are reproduced in Fig. 1A for a nerve taken from a guinea-pig 33 days after injection. This animal had developed weakness in all four limbs. The recordings from the peroneal nerves are nevertheless indistinguishable from those obtained from untreated control animals in regard to maximal conduction velocity and the amplitude of the potential was not obviously reduced. Fast repetitive stimulation caused only small changes in the form of the action potential, as in Figure $2 \mathrm{~A}$. This nerve contained several small foci of cellular infiltration and a small proportion of fibres showing myelin degeneration when stained by the Marchi method.

Eight nerves showing a mixture of normal and abnormal responses were obtained. The action potentials of Fig. 1B were recorded from a peroneal nerve of a guinea-pig injected 55 days previously. This animal had weakness in the hind limbs that was becoming more severe. The recordings show a much greater attenuation and dispersion of the action potential with distance of conduction than those in Fig. 1A, and it is difficult to measure the 


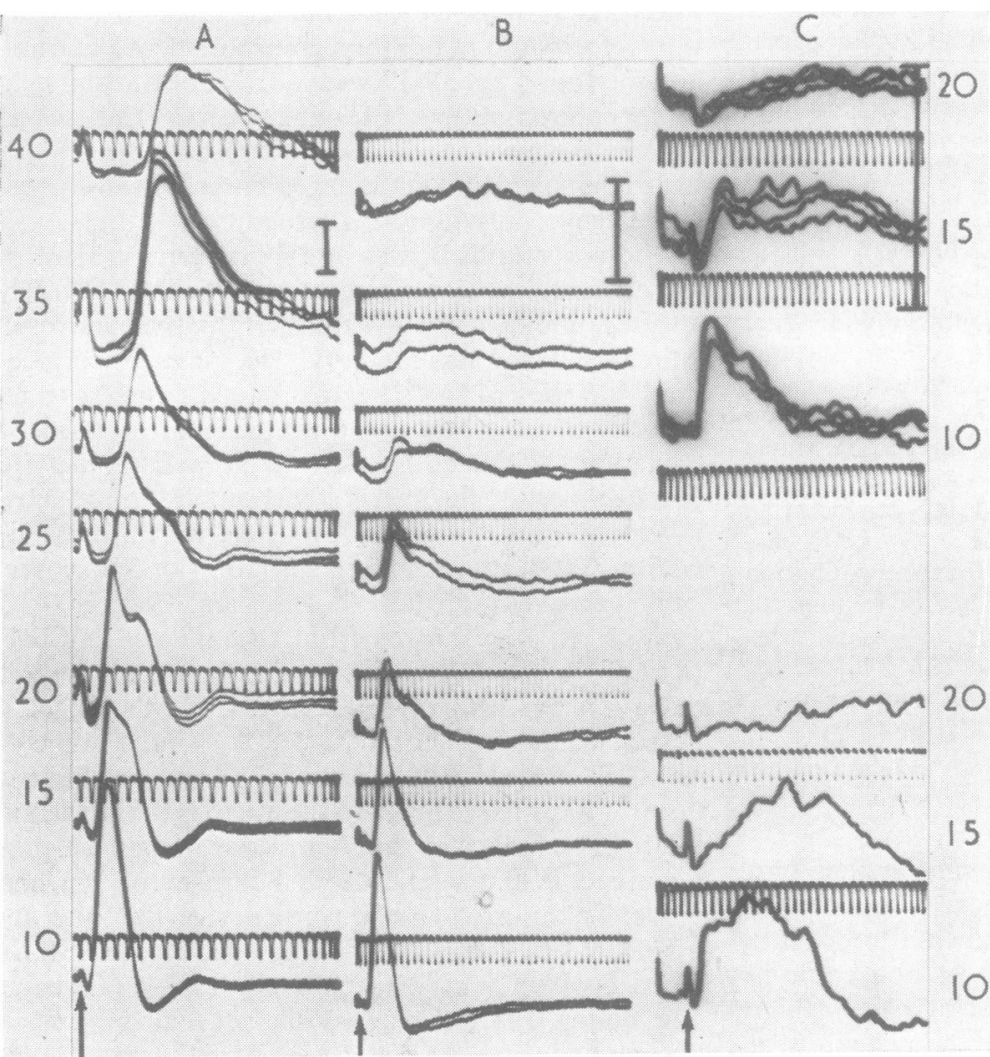

FIG. 1. Action potentials recorded from peroneal nerves of guinea-pigs with allergic neuritis. $A$ Responses of a nerve that contained some myelin degeneration, 33 days after injection. Conduction distances in millimetres in left margin, maximal velocity $50 \mathrm{~m} . / \mathrm{sec}$.; three superimposed traces. $B$ Dispersion and attenuation of the action potential in a nerve 55 days after injection. Conduction distances in millimetres in left margin, maximal velocity $55 \mathrm{~m}$. $/ \mathrm{sec}$., two superimposed traces. $C$ Extreme dispersion and attenuation of action potentials in a severely affected nerve 44 days after injection. Conduction distances in millimetres in right margin for two positions of stimulation, maximal velocity less than $10 \mathrm{~m} . / \mathrm{sec}$.; single traces or four superimposed traces. All records were photographed at a repetition rate of $1 / \mathrm{sec}$, the time marker is $10 \mathrm{kc}$./sec. throughout, the deflection produced by $1 \mathrm{mV}$ is shown by the vertical bar at the top of each column, and the position of the stimulus artefact is indicated by the arrow at the foot of each column.

FIG. 2. The effect of repetitive stimulation on the action potentials recorded from the peroneal nerves of guinea-pigs with allergic neuritis. Records are shown (1) before repetitive stimulation, (2) immediately after two minutes' stimulation at 368/sec., and (3) after five minutes' rest $(A$ and $B)$ or seven minutes $(C)$. The columns $A, B$, and $C$ and all other conditions are as in Figure 1. The conduction distances were $20 \mathrm{~mm}$. (A), $25 \mathrm{~mm}$. (B), and $10 \mathrm{~mm}$. (C).

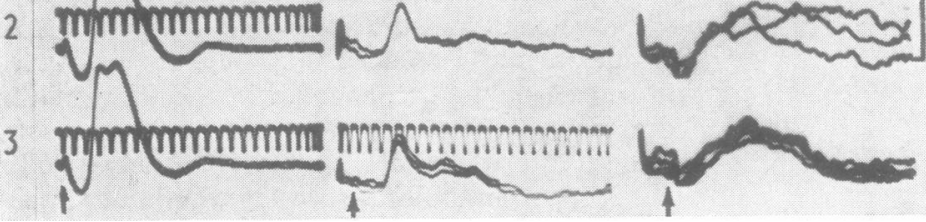

latency beyond $30 \mathrm{~mm}$. However, from 10 to $30 \mathrm{~mm}$. the latencies of the first peak are proportional to distance and indicate a maximal conduction velocity of $55 \mathrm{~m}$. $/ \mathrm{sec}$., which is not very different from the average value $(64.6 \mathrm{~m}$. $/ \mathrm{sec}$.). The small second peak seen at 20,25 , and $30 \mathrm{~mm}$. has a conduction velocity of about $30 \mathrm{~m}$./sec., and a stimulus threshold close to that of the first peak. The action potentials of normal peroneal nerves often show signs of a second $\vec{D}$ peak, so this peak could not be attributed to damaged fibres without further evidence.

It was found that after fast repetition of the stimu- $\sigma$ lus, the first peak in the action potential was little $N$ affected while the second peak was depressed (Fig. N 2B). The latter recovered within five minutes when 0 the stimulation was discontinued and showed much 
greater changes than control nerves. This nerve thus gave evidence of a normally fast component that could support repetitive responses but was dispersed and attenuated abnormally rapidly, and a slower component that was abnormally depressed by repetitive stimulation. The rapid dispersion of the action potential was not due to damage localized at the further end of the nerve from the stimulating electrodes, for a similar progressive dispersion was seen when the nerve was stimulated at this further end.

Marchi-stained sections of this nerve contained scattered foci of degenerating myelin, but it was not clear that it was more severely affected than the nerve described above.

Only three nerves showing wholly abnormal responses were obtained. The records in Fig. $1 \mathrm{C}$ were taken from a peroneal nerve of a severely affected guinea-pig 44 days after injection. The amplitude of the action potential was greatly reduced and at a conduction distance of only $10 \mathrm{~mm}$. the dispersion of the action potential is clearly much greater than normal. No definite latency could be measured beyond $15 \mathrm{~mm}$. These effects occurred at all positions of stimulation and recording along the nerve trunk, and were not due to any one particular block (see Fig. 1C). The maximal conduction velocity was not more than $10 \mathrm{~m}$./sec. After fast repetitive stimulation, the response was depressed (Fig. 2C) and did not recover completely even after seven minutes' rest. It was not possible to record from fibres of this conduction velocity in the normal nerve without removal of the epineurium, and since this recording was obtained without desheathing the nerve, it appears probable that it represents conduction in abnormal fibres rather than in surviving slow normal fibres.

This nerve contained large patches of intense cellular infiltration and myelin degeneration that must have involved a high proportion of the fibres present.

IDENTIFICATION OF NERVE FIBRES SHOWING POSTTETANIC DEPRESSION The finding that the amplitude of a maximal action potential is much reduced following a high frequency tetanus of only two minutes' duration in a severely affected nerve is as striking a departure from normality as is the fall in maximal conduction velocity and the dispersion of the action potential. It therefore seemed necessary to survey the various kinds of normal, degenerating, or regenerating nerve fibres for other examples of post-tetanic depression.

Experiments were performed on peroneal nerves that had been cut in the upper thigh just below the sciatic notch 48 or 53 hours previously. The action potentials in these nerves were much reduced in amplitude as compared with the normal side, indicat- ing failure of conduction in a proportion of the degenerating nerve fibres. However, a tetanus of $368 / \mathrm{sec}$. for two minutes, or even for 20 minutes, caused only a slight rounding of the contours of the alpha-beta action potential (Fig. 3A). This effect was only just perceptible and can in no way account for the depressed amplitude of the post-tetanic response in allergic neuritis.

Further experiments were performed in which the peroneal nerve was allowed to regenerate following a crush just distal to the sciatic notch. Nerves removed after a survival time of 35 days showed a small action potential in the distal stump with a velocity of about $9 \mathrm{~m}$./sec., but the response was hardly affected by a high frequency tetanus of two minutes' duration (Fig. 3B).

Among normal fibres it was clear that the alphabeta deflection of the larger myelinated fibres was little affected by the standard tetanus, but the possibility remained that the smaller myelinated fibres or the non-myelinated fibres might show post-tetanic depression. The vagus and saphenous nerves of normal animals were therefore studied because these nerves contain a high proportion of small fibres. It was necessary to remove the epineurium in order to record the action potentials of the small fibres. Groups of small myelinated fibres conducting at velocities between 20 and $9 \mathrm{~m}$./sec. were tested with the standard high frequency tetanus; only a slight spreading of the action potential (Fig. 3C), which disappeared within a minute of the end of the tetanus, was seen. This behaviour contrasted with that of the nerves affected with allergic neuritis, in which the post-tetanic depression was more severe and took five minutes or more to subside (Fig. 3D).

Recordings were also made of the action potentials of non-myelinated fibres in the saphenous and vagus nerves. These potentials had a conduction velocity close to $1 \mathrm{~m}$./sec., and were often further identified by the occurrence of enhancement after a short low frequency tetanus (Fig. 3E). Enhancement was not seen in the nerves affected with allergic neuritis. The non-myelinated axon potentials were often severely depressed or abolished by the standard tetanus of $368 / \mathrm{sec}$. for two minutes (Fig. 3E). Recovery occurred after five minutes without stimulation, especially if the nerve were returned to oxygenated saline. The enhancement that occurred in most nerves after a short low frequency tetanus was converted into the depression that followed a longer and faster tetanus at a point that depended apparently on the oxygenation of the nerve. An unstimulated nerve examined in liquid paraffin within a few minutes of removal from oxygenated saline showed enhancement in response to a short tetanus at a frequency as high as $110 / \mathrm{sec}$. but after 20 minutes 


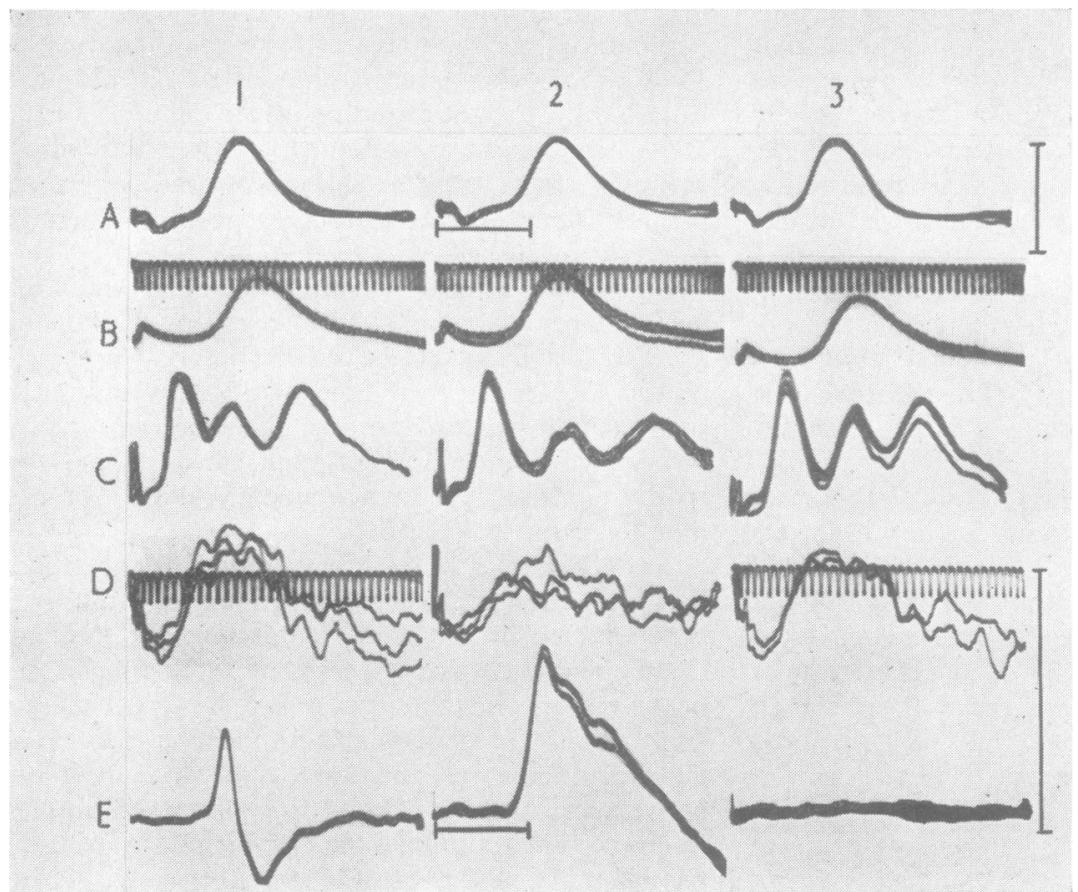

FIG. 3. The after-effects of repetitive stimulation in various classes of nerves.

A Degenerating peroneal nerve from a guinea-pig 53 hours after being cut at the hip. (1) Before, and (2) after $\vec{D}$ the standard tetanus of $368 / \mathrm{sec}$. for two min.; (3) one min. after the end of a tetanus of $368 / \mathrm{sec}$. for $20 \mathrm{minut8}$. ․․… Conduction distance $15 \mathrm{~mm}$., horizontal bar $0.3 \mathrm{m.sec}$, vertical bar $1 \mathrm{mV}$. B Regenerated peroneal nerve from $\leq a \overrightarrow{\vec{S}}$ rabbit 35 days after crushing at hip. Recordings at $10 \mathrm{~mm}$. conduction distance are shown (1) before and (2) after the standard tetanus, and (3) one min. later. Time marker $10 \mathrm{kc} . / \mathrm{sec}$, sensitivity as in A, conduction velocity $9 \mathrm{~m} . / \mathrm{sec} .+$ $C$ Small myelinated fibres in the normal cervical vagus of a rabbit. The velocities of the three peaks are 27.5, 17.5, and $9.7 \mathrm{~m} . / \mathrm{sec}$. The compound action potential is shown (1) before and (2) after the standard tetanus, and (3) one min. later. Conduction distance $20 \mathrm{~mm}$., time as in $B$, sensitivity as in A. D The peroneal nerve of a guinea-pig affected by allergic neuritis eight weeks after injection. Recordings at $25 \mathrm{~mm}$. conduction distance are shown (1) before and (2) after the stan-잉 dard tetanus, and (3) five min. later. Conduction velocity $27 \mathrm{~m} . / \mathrm{sec}$., time marker $10 \mathrm{kc} . / \mathrm{sec}$., vertical bar $1 \mathrm{mV}$.응 $E$ Non-myelinated fibres in the normal abdominal vagus of a rabbit; (1) before and (2) after a tetanus of $42 / \mathrm{sec}$. for $10 \mathrm{sec}$.,, showing enhancement, and (3) after the standard tetanus showing abolition. Velocity of spike $0.9 \mathrm{~m} . / \mathrm{sec}$., conduction $\overrightarrow{\overline{0}}$ distance $30 \mathrm{~mm}$., horizontal bar $35 \mathrm{~m} . \mathrm{sec}$., vertical bar $1 \mathrm{mV}$.

in the paraffin only low frequencies of stimulation would induce enhancement. Neither enhancement after a low frequency tetanus nor depression after a high frequency tetanus occurred invariably under the conditions of recording in mineral oil. Nevertheless, the response to tetanization of nerves severely affected with allergic neuritis has some similarity with the responses of non-myelinated fibres.

HISTOLOGICAL OBSERVATIONS The histological changes observed were similar to those reported by Waksman and Adams (1955) and therefore will not be described in detail. When stained by cresyl violet or haematoxylin and eosin, affected nerves showed focal accumulations of inflammatory cells consisting of histiocytes, lymphocytes, and other mononuclear cells. The lesions were on the average about $100 \mu$ long and elongated in the length of the nerve trunk. They occurred mainly in relation to blood vessels. On staining by the Marchi method, these foci were seen to be associated with local degeneration of the myelin sheaths. Transverse sections stained by the Weigert method revealed patchy loss of myelini sheaths (Fig. 4A), the remaining sheaths appearing normal. The silver-impregnated sections showed onlyo occasional loss of axons (Fig. 4B).

During the teasing of nerves stained with osmiumo tetroxide to obtain isolated fibres, it was evident that damage to the myelin sheaths tended to exist ino discrete patches, although occasional fibres showing damage were encountered scattered throughout the nerve trunk. When isolated fibres were examined, $\sigma$ the minimal detectable change was a widening of the nodal gap (Fig. 5B and C) in comparison with the를 

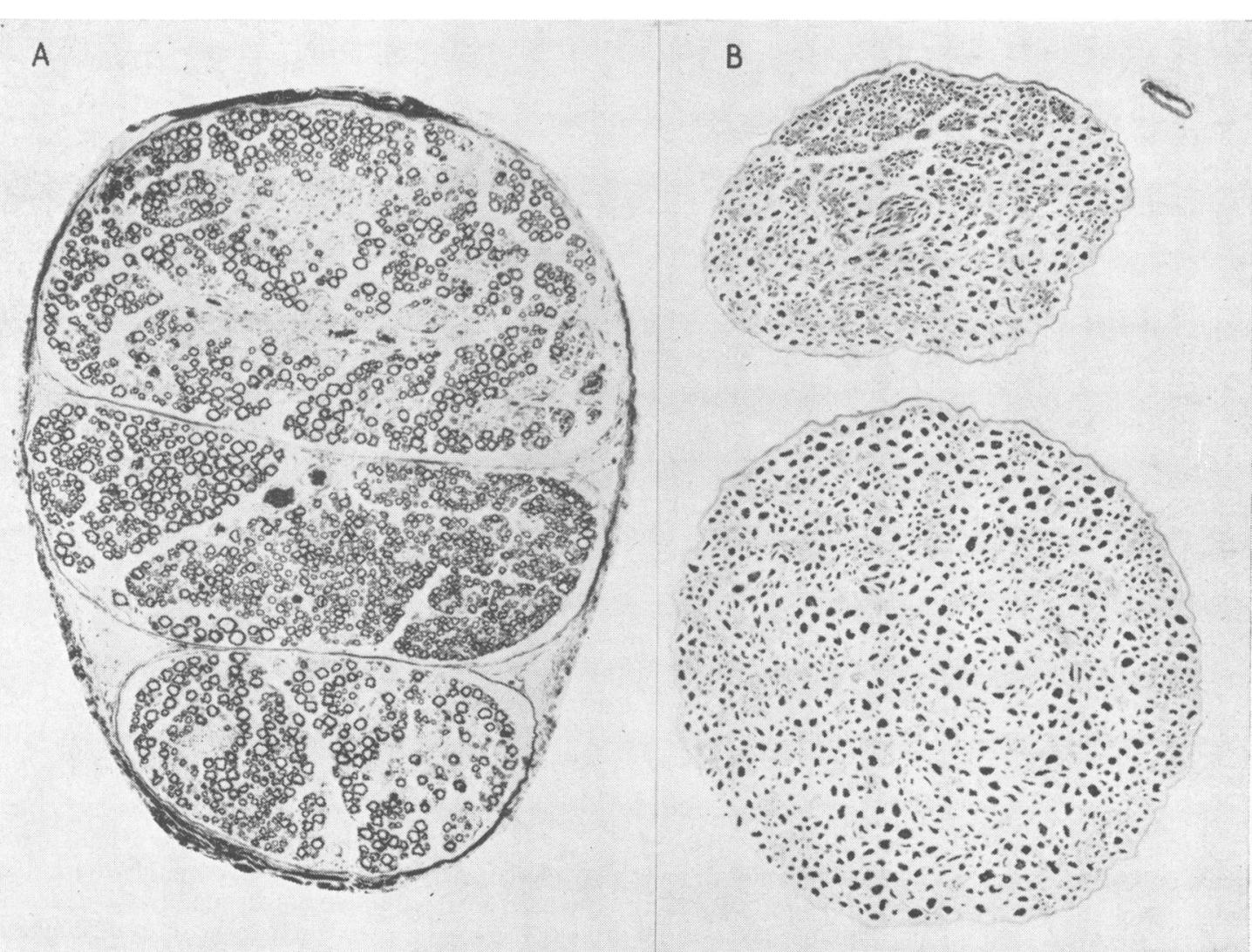

FIG. 4. A Transverse section of proximal part of peroneal nerve of a guinea-pig with allergic neuritis 34 days after injection, showing patchy loss of myelin sheaths. Weigert's haematoxylin. $\times 160 . B$ Transverse section through two fascicles from the distal part of the same nerve, stained by Glees-Marsland silver impregnation method, showing preservation of axons. $\times 220$.

normal (Fig. 5A). Other fibres showed more extensive myelin loss in the region of the nodes of Ranvier. On more severely affected fibres, loss of myelin had occurred over considerable lengths, leaving long stretches of denuded axon (Fig. 5, D-F). Comparison of the lengths of these denuded sections with the internodal length of the adjacent intact portions of the fibres indicated that they usually corresponded to the length of one internode, as was found by Lubińska (1958) for the segmental demyelination occasionally encountered in normal nerves. Sometimes the denuded stretches corresponded to two or even three internodes. Thus on one fibre, six normal internodes had an average length of $1.01 \mathrm{~mm}$. and two demyelinated portions were 1.26 and $2.12 \mathrm{~mm}$. long, corresponding to the length of one and two internodes respectively. On another fibre, a demyelinated stretch of axon $1.71 \mathrm{~mm}$. long had normal internodes 0.69 and $0.55 \mathrm{~mm}$. long on either side, the myelin thus having disappeared over the length of three internodes. Occasionally fibres were encountered with two or more demyelinated internodal segments separated by segments of apparently intact myelin.

In one animal, which had recovered after an injection made 91 days previously, some fibres that had undergone segmental demyelination showed early remyelination. In the remyelinated portions, the myelin sheath was thin and the axon narrowed as compared with the intact portions of the fibres. Internodal length was short, being 0.2 to $0.3 \mathrm{~mm}$. No abnormalities were detected in the electrical recordings of the nerves of this animal.

\section{DISCUSSION}

SEGMENTAL DEMYELINATION Segmental loss of the myelin sheath with preservation of the axon of 


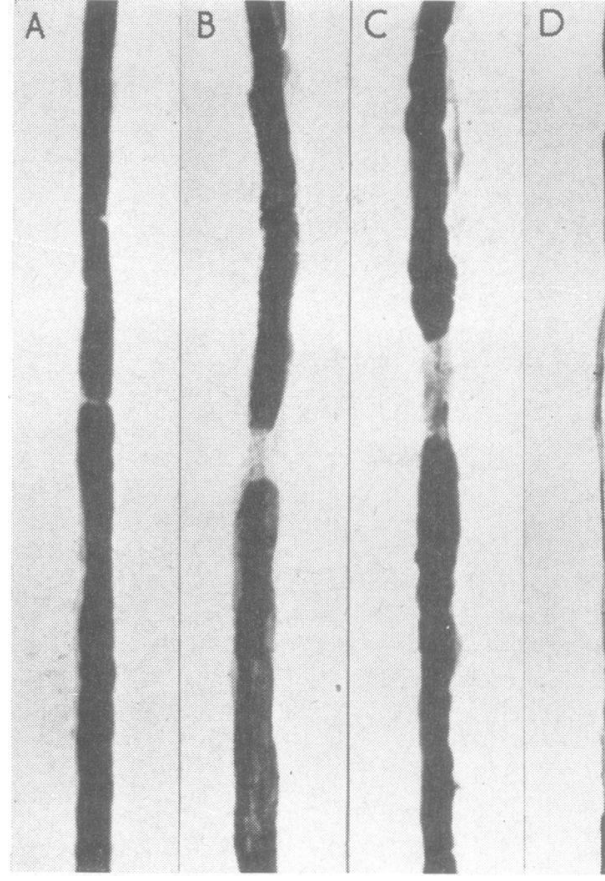

peripheral nerve fibres was first described by Gombault (1880) in experimental lead poisoning in the guinea-pig, and has been shown to be the characteristic histological lesion in diphtheritic neuritis (Waksman, Adams, and Mansmann, 1957; McDonald, 1962). Waksman and Adams (1956) found that in experimental allergic neuritis the nerve fibres showed destruction of the myelin with relative sparing of the axons, and the occurrence of segmental demyelination in this condition has been demonstrated in the present investigation by the histological examination of single fibres. The smallest recognizable lesion of the nerve fibres was the loss of myelin adjacent to the nodes of Ranvier. Although the reason for this localization is unknown, Lubińska (1959), from studies on nerve fibres above localized crush lesions, has suggested that there is a gradient of stability of the myelin sheath between the region of the Schwann cell nucleus and the ends of the internodes, the myelin of the terminal portions of the internodes being particularly vulnerable to breakdown. In the present results, on more severely affected fibres, axons were denuded of myelin for the length of several internodes.

NERVE CONDUCTION IN EXPERIMENTAL ALLERGIC NEURITIS The gross reduction in the size of the nerve action potentials in severely affected nerves indicates that many of the fibres develop a conduc-
FIG. 5. Isolated nerve fibres stained with osmium tetroxide from peroneal nerve of a guineapig with allergic neuritis 34 days after injection. A shows a node of normal appearance. In $B$ and $C$, there is loss of myelin adjacent to a node of Ranvier. In D, demyelination has occurred over the length of one internode and in $E$ and $F$, over the length of two. internodes. $A, B$, and $C \times 425$; $D \times 100 ; E$ and $F \times 42.5$. tion block. Since only occasional fibres undergoiggo Wallerian degeneration were seen, this must meagn that the conduction block is related to the demyel ating process or to axonal damage short of Walleriann degeneration. Denny-Brown and Brenner (1944) produced segmental demyelination in peripheral nerves by localized compression and found that this was associated with a conduction block. However, in addition to loss of myelin adjacent to the nodes of Ranvier or over longer stretches of the fibres, the axons also showed prominent histological changes.음 It is therefore difficult to state that the demyelination was the cause of the conduction block. Similarly, the axons in experimental allergic neuritis show histological alterations in addition to the changes in the myelin sheaths (Waksman and Adams, 1955). Tasaki (1953) has reported that in saponin-treated 3 myelinated nerve fibres, a conduction block develops. simultaneously with loss of myelin sheath insulation, 윽 but here again, it is possible that the axon membrane also becomes damaged.

The slowing of conduction in nerves affected by $D$ allergic neuritis found by ourselves and by Kaeser and Lambert (1962) indicates that not all affected N fibres suffer a conduction block but that conduction may persist in an abnormal manner. Similar findings 0 have been obtained in diphtheritic neuritis by Mc- $-\mathrm{C}$ Donald (1962) and by Kaeser and Lambert (1962) $\widetilde{\sigma}$ With regard to the cause of the slowing, Kaeser ando 
Lambert suggested that the destruction of the myelin adjacent to the nodes of Ranvier, in increasing the area of exposed axon membrane, might increase the nodal activation time by reducing the density of current flow across the membrane. Yet such marked slowing of conduction velocity is not seen in Wallerian degeneration during which no more than a $13 \%$ reduction in velocity occurs before failure of conduction sets in (Gutmann and Holubář, 1950), although retraction of myelin from the nodes of Ranvier is seen (Causey and Palmer, 1952). Alternatively, Kaeser and Lambert raised the possibility of relatively slight alterations in the myelin sheath leading to an impairment of its insulating properties. So far, electron microscope observations on the myelin sheath in allergic neuritis have not been made.

The present observations have also shown that nerves affected by allergic neuritis may exhibit posttetanic depression. The occurrence of post-tetanic enhancement was described by Brown and Holmes (1956) in non-myelinated fibres in vivo, the most effective tetanus consisting of 1,000 to 2,000 stimuli at frequencies of 50 to $100 / \mathrm{sec}$. The enhanced spike was accompanied by a large and long-lasting negative after potential (cf. Fig. 3E). Ritchie and Straub (1956) found evidence that the enhancement was due to the simultaneous recording of the negative after potential in many fibres in which the shorter action potential spike was asynchronous owing to dispersion with conduction distance. The negative after potential was accompanied, and possibly caused by, a hyperpolarization of the axon of about $4 \mathrm{mV}$. Similar enhancement of the action potential can be produced by externally applied hyperpolarizing currents, but too great a current causes anodal block of conduction (Brown and Holmes, 1956). It is thus possible that the standard tetanus $(44,000$ volleys) used in the present work would so raise the polarization of a nonmyelinated axon as to block conduction. A further possibility is that the locally available concentration of oxygen is depleted by intense activity under the anoxic conditions of recording in mineral oil. Other metabolites are depleted, for Greengard and Straub (1959) found a $17 \%$ fall in creatine phosphate and A.T.P. after only 750 impulses in mammalian C fibres. The reason why in recordings made in vitro enhancement (Brown and Holmes, 1956) or depression' do not invariably occur after appropriate tetanization is unknown. However, enhancement and severe depression never occur in myelinated fibres, and their presence therefore gives a useful indication of activity in non-myelinated fibres.

Causey and Stratmann (1954) have demonstrated post-tetanic depression in rabbit tibial nerves during Wallerian degeneration 48 hours after cutting the nerves proximally. They used a tetanus of 600 stimuli per second for two hours, however, involving one hundred times more stimuli than the standard tetanus used in the present work. Our finding that the action potential of degenerating guinea-pig nerves is not depressed by tetanization at $368 / \mathrm{sec}$. for two minutes does not therefore conflict in any way with this earlier finding. It does mean, however, that any degenerating axons still able to conduct in a nerve affected by allergic neuritis cannot be responsible for the observed post-tetanic depression.

Depression was not seen after applying the standard tetanus to regenerating nerve or to normal myelinated nerve fibres, both large and small. This means that post-tetanic depression must be a property peculiar to fibres affected by allergic neuritis, unless conduction in non-myelinated axons is in some way involved. Our results cannot be due to conduction in normal non-myelinated axons of less than $1 \mu$ diameter because the velocities recorded were much greater than $1 \mathrm{~m}$./sec., there was no posttetanic enhancement, and the recordings were made without removing the epineurium as is necessary for recording the action potential of normal non-myelinated fibres. If, however, the action potentials in some of the myelinated fibres were able to continue over the non-myelinated axon in a focus of allergic neuritis and excite the myelinated portion of the fibre on the other side of the lesion, then these nonmyelinated links could cause the whole fibre to show post-tetanic depression due to blockage of conduction in one or more of the foci between the recording and stimulating electrodes. This hypothesis requires that the difference between normal myelinated and nonmyelinated fibres in regard to post-tetanic depression is due to the difference in membrane area exposed to the action potential, rather than to a basic difference of behaviour of the membrane. Normal myelinated fibres do show post-tetanic after potentials similar to but smaller than those of non-myelinated fibres (Gasser and Grundfest, 1936), and current views of the origin of the negative after potentials in nonmyelinated fibres indicate that demyelination would increase the negative after potential in myelinated fibres should they remain capable of conducting (Douglas and Ritchie, 1962).

Myelinated fibres containing a non-myelinated link (Fig. 5, D-F) would not be expected to show enhancement of the action potential after a brief tetanus, because it has been demonstrated that in non-myelinated fibres this requires a length of several millimetres to become appreciable (Ritchie and Straub, 1956). Even in the most severely affected nerves, foci of demyelination occupy a small part of the total volume of the nerve trunk, so that the remaining myelinated axons must be responsible for most of the response recorded. Post-tetanic depres- 
sion in allergic neuritis does not usually involve complete abolition of the action potential after a standard tetanus, as often happens in a non-myelinated normal nerve, but not all the myelinated fibres in a nerve affected by allergic neuritis can be expected to pass through a focus of demyelination in the $20 \mathrm{~mm}$. or so between the stimulating and recording electrodes.

Conduction over non-myelinated links could also explain the slowing of conduction velocity. If a normal non-myelinated axon of $1 \mu$ diameter conducts at $1 \mathrm{~m}$./sec., a similar non-myelinated axon of $9 \mu$ diameter might be expected to conduct at $3 \mathrm{~m}$./sec. (Pumphrey and Young, 1938; Rushton, 1951). If, then, a myelinated fibre with a conduction velocity of $60 \mathrm{~m}$. $/ \mathrm{sec}$. and an axon diameter of $9 \mu$ is demyelinated in foci of neuritis along one tenth of its length between the recording and stimulating electrodes, the overall velocity will be reduced to about $20 \mathrm{~m}$./ $/ \mathrm{sec}$, which is of the order observed. Conduction over demyelinated segments of nerve has been postulated to account for some human examples of continued vision until death from acute disseminated sclerosis, when nearly complete demyelination of the optic nerves may be discovered (see, for example, Lumsden, 1961). Moreover, Duncan (1948) found that a short length of demyelination caused by slow compression due to an inelastic ligature placed around growing nerves resulted in little paralysis or muscular wasting, and postulated that some conduction must continue across the demyelinated region. More recently, Lehmann and Ule (1963) have reported conduction in fibres demyelinated for a length of $2 \mathrm{~mm}$. in passing through a granuloma produced by pulling a catgut thread through the nerve. These several observations suggest that the possibility of conduction across demyelinated links in allergic neuritis should be further investigated to decide whether this, or some other unknown mechanism, is responsible for the slow conduction and post-tetanic depression.

RECOVERY AFTER SEGMENTAL DEMYELINATION Regeneration of the myelin sheath after segmental demyelination results in the formation of short 'intercalated' segments of myelin, the spacing of the nodes of Ranvier being similar to that found at the time of initial myelination during development. The myelin of the remyelinated segments is at first thin and the axon in the affected region is narrowed (Gombault, 1880; Lubińska, 1958; and present results). It would be anticipated that this would be associated with a marked slowing of conduction velocity if long enough stretches of the fibre were involved. It has not been possible to follow the abnormal responses in the late stage of recovery after allergic neuritis because severely affected $\stackrel{\mathbb{D}}{\stackrel{D}{C}}$ animals do not survive, and abnormalities are not detectable in the action potentials of nerves $\subseteq$ containing only a small proportion of affected fibres.

\section{SUMMARY}

Allergic neuritis has been produced by injection of $\overrightarrow{\vec{c}}$ heterologous peripheral nerve in 59 guinea-pigs. $\frac{\vec{\sigma}}{\sigma}$ Electrical recordings were made from sciatic nerves등 removed from affected animals and stimulated in $\overline{\bar{O}}$ vitro.

Nerves containing some myelin degeneration $\propto$ taken from mildly affected animals often showed no कs electrical abnormality. Many of the more severely $\overrightarrow{0}$ affected animals died, and recordings were obtained from only 10 affected animals.

The electrical abnormalities consisted of a con- $\frac{2}{2}$ duction block in some fibres, others exhibiting a slowing of conduction velocity which resulted in an excessive dispersion in time of the action potential. iv Repetitive stimulation in the more severely affected $\vec{*}$ nerves at $368 / \mathrm{sec}$. for only two minutes depressed the action potential for about five minutes.

Histologically, the nerve trunks showed foci of $\rightarrow$ cellular infiltration and demyelination. Some singere fibres teased from nerves fixed in osmium tetroxi\& showed widening of the nodal gap, while in others $\vec{c}$ there was segmental demyelination over one more internodes with normally myelinated internodes on either side. Only occasional fibres showing Wallerian degeneration were seen.

During Wallerian degeneration nerves do not show so great a slowing of conduction velocity, nor so much dispersion and post-tetanic depression as $\varrho$ nerves affected by allergic neuritis. Among normal or $\vec{\overrightarrow{ }}$ regenerating nerves, only non-myelinated axons 3 showed comparable post-tetanic depression, although this did not always occur.

The hypothesis is examined that the action potential is conducted across some of the demyelinated internodes to excite the myelinated fibre on the other 0 side. This would explain the slowing of conduction and the post-tetanic depression, but another pos- $\delta$ sibility is that allergic neuritis confers these special properties on affected nerves by some unknown 0 mechanism.

We are indebted to Dr. A. J. Buller, Professor R. W. Gilliatt, and Professor B. Katz, F.R.S., for helpful criti-o cism of the manuscript. Part of this work was performed $N$ while one of us (P.K.T.) held an appointment in the Department of Medicine at the Montreal General $\omega$ Hospital, and we wish to thank Professor D. G. Cameron for providing generous facilities. 
REFFRENCES

Bannister, R. G., and Sears, T. A. (1962). J. Neurol. Neurosurg. Psychiat., 25, 321.

Brown, G. L., and Holmes, O. (1956). Proc. roy. Soc. B, 145, 1. Causey, G., and Palmer, E. (1952). Ibid., 139, 597.

- and Stratmann, C. J. (1954). J. Physiol. (Lond.), 123, 234

Cerra, D., and Johnson, E. W. (1961). Arch. phys. Med., 42, 159.

Denny-Brown, D., and Brenner, C. (1944). Arch. Neurol. Psychiat. (Chic.), 51, 1 .

Douglas, W. W., and Ritchie, J. M. (1962). Physiol. Rev., 42, 297.

Duncan, D. (1948). J. Neuropath. exp. Neurol., 7, 261.

Fernand, V. S. V., and Young, J. Z. (1951). Proc. roy. Soc. B, 139, 38.

Gasser, H. S., and Grundfest, H. (1936). Amer. J. Physiol., 117, 113.

Gombault, A. (1880). Arch. Neurol. (Paris), 1, 11 and 177.

Greengard, P., and Straub, R. W. (1959). J. Physiol. (Lond.), 148, 353.

Gutmann, E., and Holubár., J. (1950). J. Neurol, Neurosurg, and Psychiat., 13, 89.

- , and Sanders, F. K. (1943). J. Physiol. (Lond.), 101, 489.

Kaeser, H. E. (1962). Dtsch. Z. Nervenheilk., 183, 268.
- , and Lambert, E. H. (1962). Electroenceph. clin. Neurophysiol., Suppl. 22, 29.

Lambert, E. H., (1962). Ibid., Suppl. 22, 9.

Lehmann, H. J., and Ule, G. (1963). In Abstracts of Communications, International Meeting on Electromyography, Copenhagen, 1963.

Lubińska, L. (1958). Acta Biol. exp., (Warszawa) 18, 117.

- (1959). J. comp. Neurol., 113, 315.

Lumsden, C. E. (1961). In Scientific Aspects of Neurology, edited by H. Garland, p. 21. Livingstone, Edinburgh.

McDonald, W. I. (1962). Acta neuropath. (Berl.), 1, 425.

Pumphrey, R. J., and Young, J. Z. (1938). J. exp. Biol, 15, 453.

Ritchie, J. M., and Straub, R. W. (1956). J. Physiol. (Lond.), 134, 698.

Rushton, W. A. H. (1951). Ibid., 115, 101

Tasaki, I. (1953). Nervous Transmission, p. 78. Thomas, Springfield, Illinois.

Thomas, P. K. (1955). Proc. roy. Soc. B, 143, 380.

Waksman, B. H., and Adams, R. D. (1955). J. exp. Med., 102, 213

- - - (1956). J. Neuropath. exp. Neurol., 15, 293.

, - , and Mansmann, H. C. (1957). J. exp. Med., 105, 591. 